\title{
Detection of Chlorophyll Content of Rice Leaves by Chlorophyll Fluorescence Spectrum Based on PCA-ANN
}

\author{
Zhou Lina ${ }^{1, a}$ Cheng Shuchao ${ }^{1, b}$ Yu Haiye ${ }^{2, c}$ \\ ${ }^{1}$ College of Engineering and Technology, Jilin Agricultural University, Changchun, Jilin 130118 , \\ China \\ ${ }^{2}$ Key Laboratory of Bionic Engineering, Ministry of Education, School of Biological and Agricultural \\ Engineering, Jilin University, Changchun 130022, China \\ aemail:zhoulina976430@163.com, bemail:chengshuchao2016@126.com, cemail:haiye@jlu.edu.cn
}

Keywords: Fluorescence Spectrum; Chlorophyll; Principal Components Analysis; Artificial Neural Network

\begin{abstract}
In order to achieve the fast, nondestructive and accurate testing of rice leaves chlorophyll content, the model of detecting rice leaves' chlorophyll content was established by laser-induced chlorophyll fluorescence (LICF) spectroscopy technology and principal components analysis (PCA) combined with artificial neural network (ANN) in the present research. For comparison, multiple linear regression (MLR) was applied too. First of all, chlorophyll fluorescence spectrum and chlorophyll content of rice leaves were measured in different growth stages. Meanwhile, PCA was used to achieve the dimension reduction on spectral information, three principal components whose variance are greater than 1 and cumulative credibility is $99.81 \%$ were extracted by this method. Furthermore, due to the correlation between the chlorophyll content of leaves and the three principal components, the three principal components were used as the inputs of ANN and MLR to establish PCA-ANN model and PCA- MLR model respectively. Prediction examinations of the two models were made based on the data measured during the same period. The results show that both PCA-ANN model and PCA- MLR model can complete the forecast on the chlorophyll content, but the result of PCA- ANN model is better than the result of PCA- MLR and the minimum of its relative prediction error is $2.62 \%$, as the maximum is $4.83 \%$.
\end{abstract}

\section{Introduction}

Chlorophyll is an essential substance which make plants convert light energy into chemical energy. It's directly related to the photosynthetic rate and primary productivity of plants, and can also be used as a diagnostic indicator of environmental stress to plant and nitrogen fertilizer application[1-4]. Chlorophyll fluorescence is an ideal probe of chlorophyll status, which is characterized by rapid, accurate and non destructive[5]. When plants are affected by the change of external environment, the chlorophyll content and photosynthetic capacity of leaves will change, and the chlorophyll fluorescence spectrum will change with them too. Therefore, it has become an important research content of the plant growth condition to detect the chlorophyll content of plants by using chlorophyll fluorescence spectroscopy technology[6-8].

Chlorophyll fluorescence technique includes active fluorescence technique and passive fluorescence technique. When study on the photosynthetic function of plant canopy and primary productivity, passive fluorescence technique is often applied[9-10], but the active fluorescence technique has more advantages in the detection of plant leaves or other organs. Mauromicale $G$ studied the chlorophyll content and fluorescence characteristics of potato leaves with different nitrogen levels, different gene types and different growth stages[11]. Gamon J.A studied on leaf pigment content and activity by using leaf reflectance index[12].Anatoly A and Lichtenthaler $\mathrm{H}$. K used red and far red area chlorophyll fluorescence intensity as a measure to measure the chlorophyll content of plant leaves[8, 13-14].In the study, the fluorescence characteristics of one or several specific wavelengths and the relationship between them were used to study the chlorophyll content 
and predict the chlorophyll content, and the modeling information sources and modeling methods are relatively simple, and the prediction accuracy has yet to be improved. In this paper, based on the analysis of the living leaf total chlorophyll fluorescence spectral information on the basis of all band, the detection of chlorophyll content was attempted by using active fluorescence technique.

\section{Test Materials and Methods}

In June 28, 2015, July 21st and August 25th at $10 \mathrm{am}$, the experiment was completed in the experiment field of Changchun Agricultural Science Research Institute of Jilin province $\left(44^{\circ} 50^{\prime}\right.$ $\mathrm{N}, 125^{\circ} 18^{\prime} \mathrm{E}$ ) . The variety of rice is “Chunxiang No. 1 ", in the experiment field, pick up the 5 areas that grew evenly and managed normally, select 8 healthy rice flag leaf in each area as the measuring object, and the weather is fine in the survey. The chlorophyll fluorescence spectrum and the relative content of chlorophyll in rice leaves were obtained.

\section{Measurement of the relative content of chlorophyll}

Measurement of the relative chlorophyll content of rice leaves by hand held SPAD-502 chlorophyll meter. At the time of measurement, avoid main veins, measure the same position to chlorophyll fluorescence spectrum, measure three times synchronously and take the average value.

\section{Measurement of chlorophyll fluorescence spectra of rice leaves}

The excitation light source was derived from MBL-III-473nm type solid laser produced by Changchun Institute of Optics, Fine Mechanics and Physics, Chinese Academy of Sciences, and the excitation light intensity was selected to use 7.5mw[15]. AvaSpec-2048-USB2 type fiber optic spectrometer produced in Holland was used as fluorescence receiving device, and it's measured spectral band is $360 \sim 1100 \mathrm{~nm}$, the resolution is $2.1 \mathrm{~nm}$. During the measurement, the excitation light and the fluorescence detector form a 45 degree angle and close to the surface of the blade.

\section{Model establishment and result analysis}

Chlorophyll fluorescence spectrum and its principal components

The bands of chlorophyll fluorescence spectrum in 502 530nm (Green blue light), 632 830nm (red light and far red light) are affected significantly by physiological condition of leaves[5,16]. Therefore, the spectra of 502 830nm band were selected to study ((Fig. 1). In order to satisfy the calibration set and validation set containing different growth stages of the leaves. Half of the collection of each test constitute calibration set, the other half of the data constitutes validation set. The principal components analysis (PCA) was used to achieve the dimension reduction on spectral information of the calibration set, extract effective feature information and get the main composition and the cumulative contribution rate. The cumulative contribution rate of the 15 main components is reaching $100 \%$, and the variance of the top three is greater than or equal to 1 , the cumulative contribution rate of them is $99.81 \%$ (Table 1). Therefore, the top 3 principal components are selected to replace all the spectral data as the prediction model input values to predict chlorophyll content of rice leaves. 


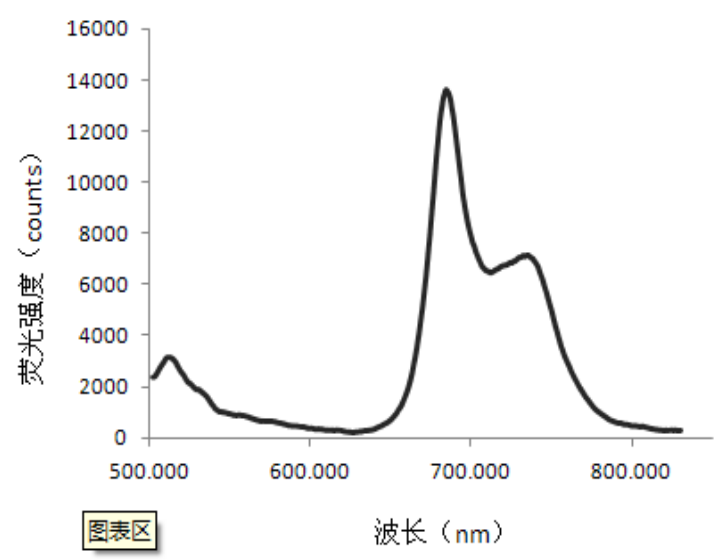

Fig.1 Laser- induced chlorophyll fluorescence spectrum of rice leaves

Table.1 Reliabilities and total variance of principal components of chlorophyll fluorescence spectrum

\begin{tabular}{cccccc}
\hline $\begin{array}{c}\text { Principal } \\
\text { component }\end{array}$ & $\begin{array}{c}\text { Variance of } \\
\text { principal } \\
\text { component } \\
\text { scores }\end{array}$ & $\begin{array}{c}\text { Cumulative } \\
\text { contribution } \\
\text { rate/\% }\end{array}$ & $\begin{array}{c}\text { Principal } \\
\text { component }\end{array}$ & $\begin{array}{c}\text { Variance of } \\
\text { principal } \\
\text { component } \\
\text { scores }\end{array}$ & $\begin{array}{c}\text { Cumulative } \\
\text { contribution } \\
\text { rate/\% }\end{array}$ \\
\hline PC1 & 444.548 & 77.312 & PC9 & 0.002 & 99.999 \\
PC2 & 83.811 & 91.888 & PC10 & 0.001 & 99.999 \\
PC3 & 45.560 & 99.812 & PC11 & 0.001 & 99.999 \\
PC4 & 0.819 & 99.954 & PC12 & 0.000 & 99.999 \\
PC5 & 0.187 & 99.986 & PC13 & 0.000 & 99.999 \\
PC6 & 0.047 & 99.994 & PC14 & 0.000 & 99.999 \\
PC7 & 0.011 & 99.998 & PC15 & 0.000 & 100 \\
PC8 & 0.003 & 99.998 & & & \\
\hline
\end{tabular}

ANN and MLR model establishment and forecast test

\section{Artificial Neural Network (ANN)}

The first 3 principal components obtained from the principal component analysis are used as input values of the artificial neural network, so the structure of artificial neural network is set up as: The vector number of the input layer is 3 , the hidden layer is 1 , the number of nodes is less than 50 (the number of nodes in the actual training time is 4 ), the number of nodes in the output layer is 1 (the relative chlorophyll content), and the error index is 0.001 .Using gradient descent optimization algorithm, the kinetic energy factor is set to 0.95 , and the interval offset is 0.5. Artificial neural network model is obtained.

\section{Multiple Linear Regression（MLR）}

By correlation analysis, the 3 principal components are significantly correlated with the relative chlorophyll content. Therefore, the traditional multiple linear regression analysis is used to compare with the PCA-ANN model. Using SPSS statistical analysis software, the regression analysis equation is:

$$
\mathrm{Y}=2.484 \mathrm{X}_{1}+6.192 \mathrm{X}_{2}+5.18 \mathrm{X}_{3}+36.741
$$

$\mathrm{X}_{1}$-First principal component ; $\mathrm{X}_{2}$-Second principal component ; $\mathrm{X}_{3}$-Third principal component 
Comparison of model prediction results

The PCA-ANN model and PCA-MLR model were tested by using the data of the validation set. Table 2 is the comparison of prediction results of two algorithm models for the relative chlorophyll content of rice leaves.

Table.2 Comparison of the prediction capabilities of PCA-ANN and PCA-MLR

\begin{tabular}{cccccc}
\hline \multirow{2}{*}{$\begin{array}{c}\text { Measurement } \\
\text { Date }\end{array}$} & Measured & Average & Forecast & Relative & Forecast \\
\cline { 3 - 6 } & Values & $\begin{array}{c}\text { Average } \\
\text { Values }\end{array}$ & Error $\%$ & $\begin{array}{c}\text { Average } \\
\text { Values }\end{array}$ & Relative Error \\
& & 49.962 & 2.88 & 48.962 & 4.83 \\
\hline 6月28日 & 51.445 & 36.285 & 9.14 & 38.890 & 2.62 \\
7月21日 & 39.935 & 33.170 & 9.01 & 34.698 & 4.82 \\
8月25日 & 36.456 & & & & \\
\hline
\end{tabular}

As can be seen from the table 2, under the premise of PCA conditions, both of MLR and ANN can detect the chlorophyll content in rice leaves. Except for June 28th, all the PCA-ANN detection relative errors were less than PCA-MLR. On the whole, the average prediction relative errors of PCA-ANN models on chlorophyll content during the three growth stages of rice are less than 5\% and its detection effect is better than PCA-MLR.

\section{Conclusion}

In this paper, the nondestructive detection of chlorophyll content in rice leaves was studied by using the technique of chlorophyll fluorescence spectrum analysis. The method of principal component analysis was used to reduce the dimension of the chlorophyll fluorescence spectrum. By correlation analysis, the relationship between the principal components and the chlorophyll content was determined. According to the variance and cumulative contribution rate, the first 3 principal components were combined with ANN and MLR to model. The results showed that the detection effect of PCA-ANN was superior to that of PCA-MLR, and it could be used as a model for the detection of chlorophyll content of rice leaves. The model can effectively extract feature information, avoid the problems of too many of independent variables and multiple correlation between variables caused by the subjective extraction of spectral characteristic information. And its prediction accuracy is better, which provides a new method for the nondestructive detection of chlorophyll content in plant leaves.

\section{Acknowledgement}

In this paper, the research was sponsored by the National High Technology Research and Development Program of China (Project No. 2013AA103005-04) and Youth Fund Project of Jilin Agricultural University (Project No. 2015004).

\section{References}

[1]Curran P J, Dungan J L, Gholz H L. Exploring the relationship between reflectance red edge and chlorophyll content in slash pine[J].Tree Physiology, 1990,7: 33 48.

[2]Merzlyak M N, Gitelson A A, Chivkunova O B, et al. Nondestructive optical detection of pigment changes during leaf senescence and fruit ripening[J].Physiologia Plantarum, 1999,106(1): 135 141. 
[3]Anatoly A G, Yuri G, Mark N M. Relationships between leaf chlorophyll content and spectral reflectance and algorithms for non-destructive chlorophyll assessment in higher plant leaves[J].Journal of Plant physiology, 2003,160(3):271 282.

[4]Filella I, Serrano I, Serra J, et al. Evaluating wheat nitrogen status with canopy relfectance indices and discriminant analysis[J].Crop Science of America, 1995,35(5): 1400 1405.

[5]Schreiber U, Bilger W, Neubauer C. Chlorophyll fluorescence as a non-destructive indicator for rapid assessment of in vivo photosynthesis[J].Ecological Studies, 1994,100(1):49 70.

[6]Khaleghi E, Arzani K, Moallemi N, et al. Evaluation of chlorophyll content and chlorophyll fluorescence parameters and relationships between chlorophyll $\mathrm{a}$, b and chlorophyll content index under water stress in Olea europaea cv[J].Dezful. World Academy of Science, Engineering and Technology, 2012,68:1154 1157.

[7]Xu Xingang, Zhao Chunjiang, Wang Jihua, et al. Study on relationship between new characteristic parameters of spectral curve and chlorophyll content for rice[J].Spectrocopy and Spectral analysis, 2011, 31(1)188 191.

[8]Gitelson A A, Buschmann C, Lichtenthaler H K. The chlorophyll fluorescence tatio F735/F700 as an accurate measure of the chlorophyll content in plants[J].Remote Sensing of Environment, 1999,69(3):296 302.

[9]Cheng Zhanhui, Liu Liangyun. Estimating light-use efficiency by the separated Solar-induced chlorophyll fluorescence from canopy spectral data[J].Journal of Remote sensing, 2010,14(2):356 363.

[10]Gilbert M, Domin A, Becker A, et al. Estimation of primary productivity by chlorophyll a in vivo fluorescence in freshwater phytoplankton[J].Photosynthetica, 2000,38(1):111 126.

[11]Mauromicale G, Ieana A, Marchese M. Chlorophyll fluorescence and chlorophyll content in field-grown potato as affected by nitrogen supply, genotype, and plant age[J].Photosynthetica, 2006, 44(1):76 82.

[12]Gamon J A, Surfus J S. Assessing leaf pigment content and activity with a reflectometer[J].New Phytologist, 1999,143(1):105 117.

[13]Lichtenthaler $\mathrm{H}$ K. Chlorophyll fluorescence signatures of leaves during the autumnal chlorophyll breakdown[J].Journal of Plant Physiology, 1987, 131(1):101 110.

[14]Lichtenthaler H K, Buschmann C. Chlorophyll fluorescence spectra of green bean leaves[J].Journal of Plant Physiology, 1987,129:137 147.

[15]Yu Haiye, Yang Haoyu, Zhang Lei, et al. Relationship between laser-induced chlorophyll fluorescence intensity and laser power[J].Transactions of the CSAE, 2009,25(Supp.2):245 249.

[16]Yang Haoyu, Yu haiye, Liu Xu, et al. Diagnosis of cucumber diseases and insect pests by fluorescence spectroscopy technology based on PCA-SVM[J]. Spectroscopy and Spectral Analysis, 2010,30(11)3018 3021. 